\title{
NEW EXACT TRAVELING WAVE SOLUTIONS TO THE (2+1)-DIMENSIONAL CHIRAL NONLINEAR SCHRÖDINGER EQUATION
}

\author{
Hadi Rezazadeh ${ }^{1}$, Muhammad Younis ${ }^{2}$, Shafqat-Ur-Rehman ${ }^{2}$, \\ Mostafa Eslami ${ }^{3, *}$, Muhammad Bilal ${ }^{2}$ and Usman Younas $^{2}$
}

\begin{abstract}
In this research work, we successfully construct various kinds of exact traveling wave solutions such as trigonometric like, singular and periodic wave solutions as well as hyperbolic solutions to the (2+1)-dimensional Chiral nonlinear Schröginger equation (CNLSE) which is used as a governing equation to discuss the wave in the quantum field theory. The mechanisms which are used to obtain these solutions are extended rational sine-cosine/sinh-cosh and the constraint conditions for the existence of valid solutions are also given. The attained results exhibit that the proposed techniques are a significant addition for exploring several types of nonlinear partial differential equations in applied sciences. Moreover, 3D, 2D-polar and contour profiles are depicted for showing the physical behavior of the reported solutions by setting suitable values of unknown parameters.
\end{abstract}

Mathematics Subject Classification. 39A14-83C15-35C08.

Received April 22, 2020. Accepted January 3, 2021.

\section{INTRODUCTION}

During the past few decades, nonlinear partial differential equations (NLPDEs) have gained great attention to scientific societies. Diverse complex nonlinear physical phenomena such as fluid mechanics, plasma physics, solid-state physics, chemical physics, geochemistry, optical fiber, quantum field theory, mathematical biology, etc., are modeled in the form of NLPDEs. To see the behavior of these intricate phenomena, it is necessary to find the solution of governing eqaution. For this, we need to extract the exact solution of the NLPDEs. Finding the analytic solutions of NLPDEs has the importance to discuss the stability of numerical solutions and also the development of a wide range of new mathematical solvers to simplify the routine calculation. Therefore, the foremost concern for the researchers is to find the exact solutions of NLPDEs. For this sake, various powerful techniques have been developed for finding the analytic solutions of NLPDEs by using different symbolic computation like Mathematica, Matlab, and Maple [2, 3, 6, 8, 11, 13-16, 18, 21, 22, 24-27, 29-36]. The study of quantum field theory is still booming, as the uses of its mechanism to many physical problems.

Keywords and phrases: Exact solutions, (2+1) CNLSE, extended rational sine-cosine/sinh-cosh techniques.

${ }^{1}$ Faculty of Engineering Technology, Amol University of Special Modern Technologies, Amol, Iran.

2 Punjab University College of Information Technology, University of the Punjab, Lahore 54000, Pakistan.

3 Department of Mathematics, Faculty of Mathematical Sciences, University of Mazandaran, Babolsar, Iran.

* Corresponding author: meslami.edu@gmail.com

(C) The authors. Published by EDP Sciences, 2021 
The quantum hall effect is a quantum-mechanical version of the hall effect that can be observed in a twodimensional electron systems subject to strong magnetic fields and low temperatures. The hall conductance undergoes quantum hall transitions to take quantized values at certain level. The prominent feature of the quantum hall effect is the persistence of the quantization when the electron density is varied. In two dimensions, when electrons are subjected to a magnetic field then they follow circular cyclotron orbits. When the system is treated quantum mechanically, these orbits are quantized. The Schrodinger's equation works as a practical model to determine the values of these energy levels. Quantum field theory remains one of the most dynamic areas of theoretical physics today, providing a common language to several other branches of physics. Solitary waves play an important role in the non-perturbative developments in the quantum field theory. Recently, the CNLSE has been analyzed by a number of effective approaches $[1,4,5,7,10,12,17,19,20,23,28,37]$ which provide effective outcomes in diverse areas of nonlinear sciences.

However our focus to find the exact traveling wave solutions and to demonstrate the efficiency of extended rational sine-cosine/sinh-cosh techniques schemes [9] to (2+1) CNLSE.

The (2+1)-dimensional CNLSE with constant coefficients is given by [7]

$$
i \Theta_{t}+a\left(\Theta_{x x}+\Theta_{y y}\right)+i\left(b_{1}\left(\Theta \Theta_{x}^{*}-\Theta^{*} \Theta_{x}\right)+b_{2}\left(\Theta \Theta_{y}^{*}-\Theta^{*} \Theta_{y}\right)\right) \Theta=0,
$$

where $\Theta$ is the complex function of $x$ and $t$, the first term is the evolution term, while $a$ indicates the coefficient of the dispersion term. Also $b_{1}, b_{2}$ are nonlinear coupling constant terms. This type of nonlinearity is called current density. The inverse scattering transform method cannot integrate the equation (1.1) as it fails the Painleve test of integrability. It is to be emphasized that above equation is not invariant under the Galilean transformation.

The basic feature of applied techniques is to establish some elementary relationships between NLPDEs and others simple NLODEs. It has been examined that with the aid of simple solutions and solvable ODEs, different kinds of exact traveling wave solutions of some complicated NLPDEs can be easily obtained. This is the key concept of above techniques, while the primary benefit of applying techniques is that we have achieved in a single move to get various types of new analytic solutions and provide us a guideline that how to organize these solutions. This method is very, consistent, efficient, reliable and much more practical, to obtain the wide range of traveling wave solutions for complex NLPDEs that appear in many fields like, telecommunication engineering, mathematical biology, mathematical physics, optical fiber and vice versa.

This piece of article is discussed as sequence: In Section 2, key points of the proposed methods. In Section 3, applications. Graphical representation in Section 4 and the conclusion in Section 5.

\section{KEY POINTS OF THE PROPOSED METHODS}

In this section we describe the first step of the extended rational methods for seeking the analytic solutions of PDEs

$$
F\left(u, \frac{\partial u}{\partial t}, \frac{\partial u}{\partial x}, \frac{\partial u^{2}}{\partial x}, \frac{\partial^{2} u}{\partial x^{2}}, \cdots\right)=0
$$

Where $u=u(x, t)$ is an unknown function and $F$ is a polynomial in $u$ and its partial derivatives. Suppose that

$$
u(x, y, t)=u(\xi), \quad \xi=x+c t,
$$

Then, by using (2.2), equation (2.1) can be turned into following ODE w.r.t. $\xi$

$$
G\left(u, u^{\prime}, u^{\prime \prime}, \cdots\right)=0
$$


In next we discuss the exact solutions of equation (2.3) by using the extended rational techniques.

\subsection{Extended rational sin-cos method}

According to this method, we assume that the analytic solution can be expressed in the following forms

$$
\begin{aligned}
& u(\xi)=\frac{a_{0} \sin (\eta \xi)}{a_{2}+a_{1} \cos (\eta \xi)}, \quad \cos (\eta \xi) \neq-\frac{a_{2}}{a_{1}}, \\
& u(\xi)=\frac{a_{0} \cos (\eta \xi)}{a_{2}+a_{1} \sin (\eta \xi)}, \quad \sin (\eta \xi) \neq-\frac{a_{2}}{a_{1}},
\end{aligned}
$$

Where $a_{0}, a_{1}$ and $a_{2}$ are parameters to be found in terms of the other parameters. The non-zero constant $\eta$ is the wave number. The derivatives of the predicted solutions are

$$
\begin{array}{r}
u^{\prime}(\xi)=\frac{a_{0} \mu\left[\cos (\eta \xi) a_{2}+a_{1}\right]}{\left[a_{2}+a_{1} \sin (\mu \xi)\right]^{2}}, \\
u^{\prime \prime}(\xi)=\frac{a_{0} \mu^{2} \sin (\eta \xi)\left[2 a_{1}^{2}+a_{1} \cos (\eta \xi) a_{2}-a_{2}^{2}\right]}{\left[a_{2}+a_{1} \cos (\eta \xi)\right]^{3}}
\end{array}
$$

in the first form and

$$
\begin{array}{r}
u^{\prime}(\xi)=-\frac{a_{0} \mu\left[\sin (\eta \xi) a_{2}+a_{1}\right]}{\left[a_{2}+a_{1} \sin (\eta \xi)\right]^{2}}, \\
u^{\prime \prime}(\xi)=\frac{a_{0} \mu^{2} \cos (\eta \xi)\left[2 a_{1}^{2}+a_{1} \sin (\eta \xi) a_{2}-a_{2}^{2}\right]}{\left[a_{2}+a_{1} \sin (\eta \xi)\right]^{3}} .
\end{array}
$$

in the second form. We substitute Equations (2.6) or (2.8) into the reduced form of the governing equation obtained above in equation (2.3). On collecting the same power coefficients of the $\cos ^{m}(\eta \xi)$ or $\sin ^{m}(\eta \xi)$ and equating to zero, we have a set of algebraic expression. The obtained algebraic polynomial produce the values of the coefficients involved. After determine $a_{0}, a_{1}, a_{2}, c$ and $\eta$ in terms of other parameters and substitute into equations (2.4) and (2.5), one gets solutions for equation (2.3) in rational sin-cos forms.

\subsection{Extended rational sinh-cosh method}

According to this method, which was introduced by Darvishi et al., [9], we suppose that the exact solution can be written in the following forms

$$
\begin{aligned}
& u(\xi)=\frac{a_{0} \sinh (\eta \xi)}{a_{2}+a_{1} \cosh (\eta \xi)}, \quad \cosh (\eta \xi) \neq-\frac{a_{2}}{a_{1}}, \\
& u(\xi)=\frac{a_{0} \cosh (\eta \xi)}{a_{2}+a_{1} \sinh (\eta \xi)}, \quad \sinh (\eta \xi) \neq-\frac{a_{2}}{a_{1}},
\end{aligned}
$$


Where $a_{0}, a_{1}$ and $a_{2}$ are parameters to be found in terms of the other parameters. The non-zero constant $\eta$ is the wave number. The derivatives of the predicted solutions are

$$
\begin{array}{r}
u^{\prime}(\xi)=\frac{a_{0} \eta\left[\cosh (\eta \xi) a_{2}+a_{1}\right]}{\left[a_{2}+a_{1} \sinh (\eta \xi)\right]^{2}}, \\
u^{\prime \prime}(\xi)=-\frac{a_{0} \eta^{2} \sinh (\eta \xi)\left[2 a_{1}^{2}+a_{1} \cosh (\eta \xi) a_{2}-a_{2}^{2}\right]}{\left[a_{2}+a_{1} \cosh (\eta \xi)\right]^{3}},
\end{array}
$$

in the first form and

$$
\begin{array}{r}
u^{\prime}(\xi)=\frac{a_{0} \eta\left[\sinh (\eta \xi) a_{2}-a_{1}\right]}{\left[a_{2}+a_{1} \sinh (\eta \xi)\right]^{2}}, \\
u^{\prime \prime}(\xi)=\frac{a_{0} \eta^{2} \cosh (\eta \xi)\left[2 a_{1}^{2}-a_{1} \sinh (\eta \xi) a_{2}+a_{2}^{2}\right]}{\left[a_{2}+a_{1} \sinh (\eta \xi)\right]^{3}} .
\end{array}
$$

in the second form. We substitute Equations (2.12) or (2.14) into the reduced form of the governing equation obtained above in equation (2.3). On collecting the same power coefficients of the $\cosh ^{m}(\eta \xi)$ or $\sinh ^{m}(\eta \xi)$ and equating to zero, we have a set of algebraic expression. The obtained algebraic polynomial produce the values of the coefficients involved. After determine $a_{0}, a_{1}, a_{2}, c$ and $\eta$ in terms of other parameters and substitute into equations (2.10) and (2.11), one gets solutions for equation (2.3) in rational sinh-cosh forms.

\section{Applications}

For equation (1.1), we start with the complex wave transformation:

$$
\Theta(x, y, t)=\Psi(\tau) e^{i \Phi}, \tau=\alpha x+\beta y-v t, \Phi=p x+q y+\omega t+\varphi
$$

where $c, \nu, \varphi, \omega$ and $k$ are parameters, which represent the amplitude component of the soliton, velocity of soliton, phase constant, frequency and wave number respectively. Substituting (3.1) into equation (1.1), we get the real part

$$
a\left(\alpha^{2}+\beta^{2}\right) \Psi^{\prime \prime}+2\left(p b_{1}+q b_{2}\right) \Psi^{3}-\left(a\left(p^{2}+q^{2}\right)+\omega\right) \Psi=0 .
$$

and the relation

$$
\nu=2 a(\alpha p+\beta q)
$$

from the imaginary part.

\subsection{Solutions via extended rational sine-cosine method}

Suppose that (3.2) has the solutions in the form

$$
\Psi(\tau)=\frac{a_{0} \sin (\eta \tau)}{a_{2}+a_{1} \cos (\eta \tau)}
$$


Substituting equation (3.4) and its derivative into equation (3.2) and the coefficients with the same power of $\cos (\eta \tau)^{m}$ equating to zero and resultantly, we get the following set of algebraic equations by using Mathematica:

$\cos (\eta \tau)^{2}: \quad-2 a_{0}^{2} b_{1} p-2 a_{0}^{2} b_{2} q-a a_{1}^{2} p^{2}-a a_{1}^{2} q^{2}-a_{1}^{2} \omega=0$,

$\cos (\eta \tau)^{1}: \quad a \alpha^{2} a_{1} a_{2} \eta^{2}+a a_{1} a_{2} \beta^{2} \eta^{2}-2 a a_{1} a_{2} p^{2}-2 a a_{1} a_{2} q^{2}-2 a_{1} a_{2} \omega=0$

$\cos (\eta \tau)^{0}: \quad 2 a \alpha^{2} a_{1}^{2} \eta^{2}-a \alpha^{2} a_{2}^{2} \eta^{2}+2 a a_{1}^{2} \beta^{2} \eta^{2}-a a_{2}^{2} \beta^{2} \eta^{2}-a a_{2}^{2} p^{2}-a a_{2}^{2} q^{2}-a_{2}^{2} \omega+2 a_{0}^{2} b_{1} p+2 a_{0}^{2} b_{2} q=0$.

On solving these algebraic equations, we get the following solution sets as:

Set-1

$$
\eta= \pm \sqrt{\frac{a\left(p^{2}+q^{2}\right)+\omega}{2 a\left(\alpha^{2}+\beta^{2}\right)}}, a_{0}= \pm \sqrt{\frac{a\left(p^{2}+q^{2}\right)+\omega}{-2\left(p b_{1}+q b_{2}\right)}} a_{1}, a_{1}=a_{1}, a_{2}=0
$$

\section{Set-2}

$$
\eta= \pm \sqrt{\frac{2 a\left(p^{2}+q^{2}\right)+\omega}{a\left(\alpha^{2}+\beta^{2}\right)}}, a_{0}= \pm \sqrt{\frac{a\left(p^{2}+q^{2}\right)+\omega}{-2\left(p b_{1}+q b_{2}\right)}} a_{1}, a_{1}=a_{1}, a_{2}= \pm a_{1} .
$$

For set 1 the solutions of equation (1.1) can be written as :

$$
\begin{aligned}
& \Theta_{1,1}(x, y, t)=\sqrt{\frac{a\left(p^{2}+q^{2}\right)+\omega}{-2\left(p b_{1}+q b_{2}\right)}} \tan \left[\sqrt{\frac{a\left(p^{2}+q^{2}\right)+\omega}{2 a\left(\alpha^{2}+\beta^{2}\right)}}(\alpha x+\beta y-v t)\right] e^{i(p x+q y+\omega t+\varphi) .} \\
& \Theta_{1,2}(x, y, t)=-\sqrt{\frac{a\left(p^{2}+q^{2}\right)+\omega}{-2\left(p b_{1}+q b_{2}\right)}} \tan \left[\sqrt{\frac{a\left(p^{2}+q^{2}\right)+\omega}{2 a\left(\alpha^{2}+\beta^{2}\right)}}(\alpha x+\beta y-v t)\right] e^{i(p x+q y+\omega t+\varphi)} .
\end{aligned}
$$

Similarly, for set 2 the solutions of equation (1.1) can be written as :

$$
\begin{aligned}
& \Theta_{2,1}(x, y, t)=\sqrt{\frac{a\left(p^{2}+q^{2}\right)+\omega}{-2\left(p b_{1}+q b_{2}\right)}} \frac{\sin \left[\sqrt{\frac{2 a\left(p^{2}+q^{2}\right)+\omega}{a\left(\alpha^{2}+\beta^{2}\right)}}(\alpha x+\beta y-v t)\right]}{1+\cos \left[\sqrt{\frac{2 a\left(p^{2}+q^{2}\right)+\omega}{a\left(\alpha^{2}+\beta^{2}\right)}}(\alpha x+\beta y-v t)\right]} e^{i(p x+q y+\omega t+\varphi)} . \\
& \Theta_{2,2}(x, y, t)=\sqrt{\frac{a\left(p^{2}+q^{2}\right)+\omega}{-2\left(p b_{1}+q b_{2}\right)}} \frac{\sin \left[\sqrt{\frac{2 a\left(p^{2}+q^{2}\right)+\omega}{a\left(\alpha^{2}+\beta^{2}\right)}}(\alpha x+\beta y-v t)\right]}{1-\cos \left[\sqrt{\frac{2 a\left(p^{2}+q^{2}\right)+\omega}{a\left(\alpha^{2}+\beta^{2}\right)}}(\alpha x+\beta y-v t)\right]} e^{i(p x+q y+\omega t+\varphi)} . \\
& \Theta_{2,3}(x, y, t)=-\sqrt{\frac{a\left(p^{2}+q^{2}\right)+\omega}{-2\left(p b_{1}+q b_{2}\right)}} \frac{\sin \left[\sqrt{\frac{2 a\left(p^{2}+q^{2}\right)+\omega}{a\left(\alpha^{2}+\beta^{2}\right)}}(\alpha x+\beta y-v t)\right]}{1+\cos \left[\sqrt{\frac{2 a\left(p^{2}+q^{2}\right)+\omega}{a\left(\alpha^{2}+\beta^{2}\right)}}(\alpha x+\beta y-v t)\right]} e^{i(p x+q y+\omega t+\varphi) .}
\end{aligned}
$$




$$
\Theta_{2,4}(x, y, t)=-\sqrt{\frac{a\left(p^{2}+q^{2}\right)+\omega}{-2\left(p b_{1}+q b_{2}\right)}} \frac{\sin \left[\sqrt{\frac{2 a\left(p^{2}+q^{2}\right)+\omega}{a\left(\alpha^{2}+\beta^{2}\right)}}(\alpha x+\beta y-v t)\right]}{1-\cos \left[\sqrt{\frac{2 a\left(p^{2}+q^{2}\right)+\omega}{a\left(\alpha^{2}+\beta^{2}\right)}}(\alpha x+\beta y-v t)\right]} e^{i(p x+q y+\omega t+\varphi)} .
$$

\section{OR}

Consider the equation (3.2) has solutions in the form, as

$$
\Psi(\tau)=\frac{a_{0} \cos (\eta \tau)}{a_{2}+a_{1} \sin (\eta \tau)}
$$

Substituting equation (3.11) and its derivative into equation (3.2) and the coefficients with the same power of $\sin (\eta \tau)^{m}$ equating to zero and resultantly, we get the following set of algebraic equations by using Mathematica:

$$
\begin{array}{ll}
\sin (\eta \tau)^{2}: & 2 a_{0}^{2} b_{1} p+2 a_{0}^{2} b_{2} q+a a_{1}^{2} p^{2}+a a_{1}^{2} q^{2}+a_{1}^{2} \omega=0, \\
\sin (\eta \tau)^{1}: & 2 a a_{1} a_{2} p^{2}+2 a a_{1} a_{2} q^{2}+2 a_{1} a_{2} \omega a-\alpha^{2} a_{1} a_{2} \eta^{2}-a a_{1} a_{2} \beta^{2} \eta^{2}=0, \\
\sin (\eta \tau)^{0}: & a a_{2}^{2} \beta^{2} \eta^{2}+a a_{2}^{2} p^{2}-a a_{2}^{2} q^{2}+a_{2}^{2} \omega-2 a \alpha^{2} a_{1}^{2} \eta^{2}+a \alpha^{2} a_{2}^{2} \eta^{2}-2 a a_{1}^{2} \beta^{2} \eta^{2}-2 a_{0}^{2} b_{1} p-2 a_{0}^{2} b_{2} q=0 .
\end{array}
$$

On solving these algebraic equations, we get the following solution sets as:

\section{Set-3}

$$
\eta= \pm \sqrt{\frac{a\left(p^{2}+q^{2}\right)+\omega}{2 a\left(\alpha^{2}+\beta^{2}\right)}}, a_{0}= \pm \sqrt{\frac{a\left(p^{2}+q^{2}\right)+\omega}{-2\left(p b_{1}+q b_{2}\right)}} a_{1}, a_{1}=a_{1}, a_{2}=0 .
$$

\section{Set-4}

$$
\eta= \pm \sqrt{\frac{2 a\left(p^{2}+q^{2}\right)+\omega}{a\left(\alpha^{2}+\beta^{2}\right)}}, a_{0}= \pm \sqrt{\frac{a\left(p^{2}+q^{2}\right)+\omega}{-2\left(p b_{1}+q b_{2}\right)}} a_{1}, a_{1}=a_{1}, a_{2}= \pm a_{1} .
$$

For set 3 the solutions of equation (1.1) can be written as :

$$
\begin{gathered}
\Theta_{3,1}(x, y, t)=\sqrt{\frac{a\left(p^{2}+q^{2}\right)+\omega}{-2\left(p b_{1}+q b_{2}\right)}} \cot \left[\sqrt{\frac{a\left(p^{2}+q^{2}\right)+\omega}{2 a\left(\alpha^{2}+\beta^{2}\right)}}(\alpha x+\beta y-v t)\right] e^{i(p x+q y+\omega t+\varphi)} . \\
\Theta_{3,2}(x, y, t)=-\sqrt{\frac{a\left(p^{2}+q^{2}\right)+\omega}{-2\left(p b_{1}+q b_{2}\right)}} \cot \left[\sqrt{\frac{a\left(p^{2}+q^{2}\right)+\omega}{2 a\left(\alpha^{2}+\beta^{2}\right)}}(\alpha x+\beta y-v t)\right] e^{i(p x+q y+\omega t+\varphi)} .
\end{gathered}
$$

Similarly, for set 4 the solutions of equation (1.1) can be written as :

$$
\Theta_{4,1}(x, y, t)=\sqrt{\frac{a\left(p^{2}+q^{2}\right)+\omega}{-2\left(p b_{1}+q b_{2}\right)}} \frac{\cos \left[\sqrt{\frac{2 a\left(p^{2}+q^{2}\right)+\omega}{a\left(\alpha^{2}+\beta^{2}\right)}}(\alpha x+\beta y-v t)\right]}{1+\sin \left[\sqrt{\frac{2 a\left(p^{2}+q^{2}\right)+\omega}{a\left(\alpha^{2}+\beta^{2}\right)}}(\alpha x+\beta y-v t)\right]} e^{i(p x+q y+\omega t+\varphi)} .
$$




$$
\begin{aligned}
& \Theta_{4,2}(x, y, t)=\sqrt{\frac{a\left(p^{2}+q^{2}\right)+\omega}{-2\left(p b_{1}+q b_{2}\right)}} \frac{\cos \left[\sqrt{\frac{2 a\left(p^{2}+q^{2}\right)+\omega}{a\left(\alpha^{2}+\beta^{2}\right)}}(\alpha x+\beta y-v t)\right]}{1-\sin \left[\sqrt{\frac{2 a\left(p^{2}+q^{2}\right)+\omega}{a\left(\alpha^{2}+\beta^{2}\right)}}(\alpha x+\beta y-v t)\right]} e^{i(p x+q y+\omega t+\varphi)} . \\
& \Theta_{4,3}(x, y, t)=-\sqrt{\frac{a\left(p^{2}+q^{2}\right)+\omega}{-2\left(p b_{1}+q b_{2}\right)}} \frac{\cos \left[\sqrt{\frac{2 a\left(p^{2}+q^{2}\right)+\omega}{a\left(\alpha^{2}+\beta^{2}\right)}}(\alpha x+\beta y-v t)\right]}{1+\sin \left[\sqrt{\frac{2 a\left(p^{2}+q^{2}\right)+\omega}{a\left(\alpha^{2}+\beta^{2}\right)}}(\alpha x+\beta y-v t)\right]} e^{i(p x+q y+\omega t+\varphi)} . \\
& \Theta_{4,4}(x, y, t)=-\sqrt{\frac{a\left(p^{2}+q^{2}\right)+\omega}{-2\left(p b_{1}+q b_{2}\right)}} \frac{\cos \left[\sqrt{\frac{2 a\left(p^{2}+q^{2}+\omega\right.}{a\left(\alpha^{2}+\beta^{2}\right)}}(\alpha x+\beta y-v t)\right]}{1-\sin \left[\sqrt{\frac{2 a\left(p^{2}+q^{2}\right)+\omega}{a\left(\alpha^{2}+\beta^{2}\right)}}(\alpha x+\beta y-v t)\right]} e^{i(p x+q y+\omega t+\varphi) .}
\end{aligned}
$$

\subsection{Solutions via extended rational sinh-cosh method}

Assume that the equation (3.2) has solutions of the form

$$
\Psi(\tau)=\frac{a_{0} \sinh (\eta \tau)}{a_{2}+a_{1} \cosh (\eta \tau)}
$$

Substituting equation (3.18) and its derivative into equation (3.2) and the coefficients with the same power of $\cosh (\eta \tau)^{m}$ equating to zero and resultantly, we get the following set of algebraic equations by using Mathematica:

$$
\begin{aligned}
& \cosh (\eta \tau)^{2}: \quad 2 a_{0}^{2} b_{1} p+2 a_{0}^{2} b_{2} q-a a_{1}^{2} p^{2}-a a_{1}^{2} q^{2}-a_{1}^{2} \omega=0, \\
& \cosh (\eta \tau)^{1}: \quad-a \alpha^{2} a_{1} a_{2} \eta^{2}-a a_{1} a_{2} \beta^{2} \eta^{2}-2 a a_{1} a_{2} p^{2}-2 a a_{1} a_{2} q^{2}-2 a_{1} a_{2} \omega=0, \\
& \cosh (\eta \tau)^{0}: \quad-2 a \alpha^{2} a_{1}^{2} \eta^{2}+a \alpha^{2} a_{2}^{2} \eta^{2}-2 a a_{1}^{2} \beta^{2} \eta^{2}+a a_{2}^{2} \beta^{2} \eta^{2}-2 a_{0}^{2} b_{1} p-2 a_{0}^{2} b_{2} q-a a_{2}^{2} p^{2}-a a_{2}^{2} q^{2}-a_{2}^{2} \omega=0 .
\end{aligned}
$$

On solving these algebraic equations, we get the following solution sets as:

Set-5

$$
\eta= \pm \sqrt{\frac{-\left(a p^{2}+a q^{2}+\omega\right)}{2 a\left(\alpha^{2}+\beta^{2}\right)}}, a_{0}= \pm \sqrt{\frac{a\left(p^{2}+q^{2}\right)+\omega}{2\left(p b_{1}+q b_{2}\right)}} a_{1}, a_{1}=a_{1}, a_{2}=0
$$

\section{Set-6}

$$
\eta= \pm \sqrt{\frac{-2\left(a p^{2}+a q^{2}+\omega\right)}{a\left(\alpha^{2}+\beta^{2}\right)}}, a_{0}= \pm \sqrt{\frac{a\left(p^{2}+q^{2}\right)+\omega}{2\left(p b_{1}+q b_{2}\right)}} a_{1}, a_{1}=a_{1}, a_{2}= \pm a_{1}
$$


For set 5 the solutions of equation (1.1) can be written as :

$$
\begin{gathered}
\Theta_{5,1}(x, y, t)=\sqrt{\frac{a\left(p^{2}+q^{2}\right)+\omega}{2\left(p b_{1}+q b_{2}\right)}} \tanh \left[\sqrt{\frac{-\left(a p^{2}+a q^{2}+\omega\right)}{2 a\left(\alpha^{2}+\beta^{2}\right)}}(\alpha x+\beta y-v t)\right] e^{i(p x+q y+\omega t+\varphi) .} \\
\Theta_{5,2}(x, y, t)=-\sqrt{\frac{a\left(p^{2}+q^{2}\right)+\omega}{2\left(p b_{1}+q b_{2}\right)}} \tanh \left[\sqrt{\frac{-\left(a p^{2}+a q^{2}+\omega\right)}{2 a\left(\alpha^{2}+\beta^{2}\right)}}(\alpha x+\beta y-v t)\right] e^{i(p x+q y+\omega t+\varphi)} .
\end{gathered}
$$

Similarly, for set 6 the solutions of equation (1.1) can be written as :

$$
\begin{aligned}
& \Theta_{6,1}(x, y, t)=\sqrt{\frac{a\left(p^{2}+q^{2}\right)+\omega}{2\left(p b_{1}+q b_{2}\right)}} \frac{\sinh \left[\sqrt{\frac{-2\left(a p^{2}+a q^{2}+\omega\right)}{a\left(\alpha^{2}+\beta^{2}\right)}}(\alpha x+\beta y-v t)\right]}{1+\cosh \left[\sqrt{\frac{-2\left(a p^{2}+a q^{2}+\omega\right)}{a\left(\alpha^{2}+\beta^{2}\right)}}(\alpha x+\beta y-v t)\right]} e^{i(p x+q y+\omega t+\varphi)} . \\
& \Theta_{6,2}(x, y, t)=\sqrt{\frac{a\left(p^{2}+q^{2}\right)+\omega}{2\left(p b_{1}+q b_{2}\right)}} \frac{\sinh \left[\sqrt{\frac{-2\left(a p^{2}+a q^{2}+\omega\right)}{a\left(\alpha^{2}+\beta^{2}\right)}}(\alpha x+\beta y-v t)\right]}{1-\cosh \left[\sqrt{\frac{-2\left(a p^{2}+a q^{2}+\omega\right)}{a\left(\alpha^{2}+\beta^{2}\right)}}(\alpha x+\beta y-v t)\right]} e^{i(p x+q y+\omega t+\varphi)} . \\
& \Theta_{6,3}(x, y, t)=-\sqrt{\frac{a\left(p^{2}+q^{2}\right)+\omega}{2\left(p b_{1}+q b_{2}\right)}} \frac{\sinh \left[\sqrt{\frac{-2\left(a p^{2}+a q^{2}+\omega\right)}{a\left(\alpha^{2}+\beta^{2}\right)}}(\alpha x+\beta y-v t)\right]}{1+\cosh \left[\sqrt{\frac{-2\left(a p^{2}+a q^{2}+\omega\right)}{a\left(\alpha^{2}+\beta^{2}\right)}}(\alpha x+\beta y-v t)\right]} e^{i(p x+q y+\omega t+\varphi)} . \\
& \Theta_{6,4}(x, y, t)=-\sqrt{\frac{a\left(p^{2}+q^{2}\right)+\omega}{2\left(p b_{1}+q b_{2}\right)}} \frac{\sinh \left[\sqrt{\frac{-2\left(a p^{2}+a q^{2}+\omega\right)}{a\left(\alpha^{2}+\beta^{2}\right)}}(\alpha x+\beta y-v t)\right]}{1-\cosh \left[\sqrt{\frac{-2\left(a p^{2}+a q^{2}+\omega\right)}{a\left(\alpha^{2}+\beta^{2}\right)}}(\alpha x+\beta y-v t)\right]} e^{i(p x+q y+\omega t+\varphi)} .
\end{aligned}
$$

OR

Consider the equation (3.2) has the solutions in the form as

$$
\Psi(\tau)=\frac{a_{0} \cosh (\eta \tau)}{a_{2}+a_{1} \sinh (\eta \tau)}
$$

Substituting equation (3.25) and its derivative into equation (3.2) and the coefficients, with the same power of $\sinh (\eta \tau)^{m}$ equating to zero and resultantly, we get the following set of algebraic equations by using Mathematica:

$\sinh (\eta \tau)^{2}: \quad 2 a_{0}^{2} b_{1} p+2 a_{0}^{2} b_{2} q-a a_{1}^{2} p^{2}-a a_{1}^{2} q^{2}-a_{1}^{2} \omega$, $\sinh (\eta \tau)^{1}: \quad-a \alpha^{2} a_{1} a_{2} \eta^{2}-a a_{1} a_{2} \beta^{2} \eta^{2}-2 a a_{1} a_{2} p^{2}-2 a a_{1} a_{2} q^{2}-2 a_{1} a_{2} \omega$ 
$\sinh (\eta \tau)^{0}: \quad 2 a \alpha^{2} a_{1}^{2} \eta^{2}+a \alpha^{2} a_{2}^{2} \eta^{2}+2 a a_{1}^{2} \beta^{2} \eta^{2}+a a_{2}^{2} \beta^{2} \eta^{2}+2 a_{0}^{2} b_{1} p+2 a_{0}^{2} b_{2} q-a a_{2}^{2} p^{2}-a a_{2}^{2} q^{2}-a_{2}^{2} \omega$.

On solving these algebraic equations, we get the following solution sets as:

\section{Set-7}

$$
\eta= \pm \sqrt{\frac{-\left(a p^{2}+a q^{2}+\omega\right)}{2 a\left(\alpha^{2}+\beta^{2}\right)}}, a_{0}= \pm \sqrt{\frac{a\left(p^{2}+q^{2}\right)+\omega}{2\left(p b_{1}+q b_{2}\right)}} a_{1}, a_{1}=a_{1}, a_{2}=0 .
$$

\section{Set-8}

$$
\eta= \pm \sqrt{\frac{-2\left(a p^{2}+a q^{2}+\omega\right)}{a\left(\alpha^{2}+\beta^{2}\right)}}, a_{0}= \pm \sqrt{\frac{a\left(p^{2}+q^{2}\right)+\omega}{2\left(p b_{1}+q b_{2}\right)}} a_{1}, a_{1}=a_{1}, a_{2}= \pm a_{1}
$$

For set 7 the solutions of equation (1.1) can be written as :

$$
\begin{aligned}
& \Theta_{7,1}(x, y, t)=\sqrt{\frac{a\left(p^{2}+q^{2}\right)+\omega}{2\left(p b_{1}+q b_{2}\right)}} \operatorname{coth}\left[\sqrt{\frac{-\left(a p^{2}+a q^{2}+\omega\right)}{2 a\left(\alpha^{2}+\beta^{2}\right)}}(\alpha x+\beta y-v t)\right] e^{i(p x+q y+\omega t+\varphi)} . \\
& \Theta_{7,2}(x, y, t)=-\sqrt{\frac{a\left(p^{2}+q^{2}\right)+\omega}{2\left(p b_{1}+q b_{2}\right)}} \operatorname{coth}\left[\sqrt{\frac{-\left(a p^{2}+a q^{2}+\omega\right)}{2 a\left(\alpha^{2}+\beta^{2}\right)}}(\alpha x+\beta y-v t)\right] e^{i(p x+q y+\omega t+\varphi)} .
\end{aligned}
$$

Similarly, for set 8 the solutions of equation (1.1) can be written as:

$$
\begin{aligned}
& \Theta_{8,1}(x, y, t)=\sqrt{\frac{a\left(p^{2}+q^{2}\right)+\omega}{2\left(p b_{1}+q b_{2}\right)}} \frac{\cosh \left[\sqrt{\frac{-2\left(a p^{2}+a q^{2}+\omega\right)}{a\left(\alpha^{2}+\beta^{2}\right)}}(\alpha x+\beta y-v t)\right]}{1+\sinh \left[\sqrt{\frac{-2\left(a p^{2}+a q^{2}+\omega\right)}{a\left(\alpha^{2}+\beta^{2}\right)}}(\alpha x+\beta y-v t)\right]} e^{i(p x+q y+\omega t+\varphi)} . \\
& \Theta_{8,2}(x, y, t)=\sqrt{\frac{a\left(p^{2}+q^{2}\right)+\omega}{2\left(p b_{1}+q b_{2}\right)}} \frac{\cosh \left[\sqrt{\frac{-2\left(a p^{2}+a q^{2}+\omega\right)}{a\left(\alpha^{2}+\beta^{2}\right)}}(\alpha x+\beta y-v t)\right]}{1-\sinh \left[\sqrt{\frac{-2\left(a p^{2}+a q^{2}+\omega\right)}{a\left(\alpha^{2}+\beta^{2}\right)}}(\alpha x+\beta y-v t)\right]} e^{i(p x+q y+\omega t+\varphi)} . \\
& \Theta_{8,3}(x, y, t)=-\sqrt{\frac{a\left(p^{2}+q^{2}\right)+\omega}{2\left(p b_{1}+q b_{2}\right)}} \frac{\cosh \left[\sqrt{\frac{-2\left(a p^{2}+a q^{2}+\omega\right)}{a\left(\alpha^{2}+\beta^{2}\right)}}(\alpha x+\beta y-v t)\right]}{1+\sinh \left[\sqrt{\frac{-2\left(a p^{2}+a q^{2}+\omega\right)}{a\left(\alpha^{2}+\beta^{2}\right)}}(\alpha x+\beta y-v t)\right]} e^{i(p x+q y+\omega t+\varphi)} .
\end{aligned}
$$



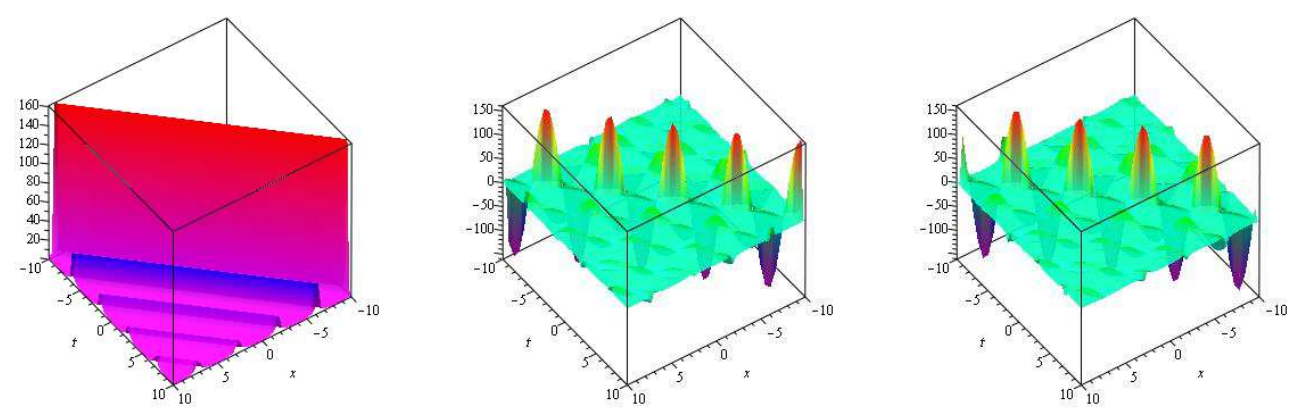

(a)

$$
\left|\theta_{2,2}\right|
$$

$\operatorname{Re}\left(\theta_{2,2}\right)$

$\operatorname{Im}\left(\theta_{2,2}\right)$
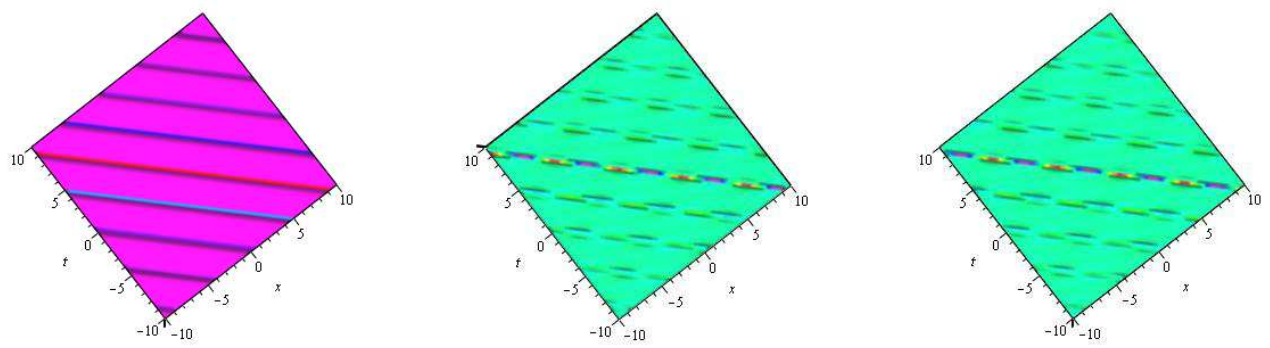

(b)

$\left|\theta_{2,2}\right|$

$R e\left(\theta_{2,2}\right)$

$\operatorname{Im}\left(\theta_{2,2}\right)$


(c)

$$
\left|\theta_{2,2}\right|
$$

$\operatorname{Re}\left(\theta_{2,2}\right)$

$\operatorname{Im}\left(\theta_{2,2}\right)$

Figure 1. (a) 3D-plot of the modulus (left), real (middle) and imaginary (right) parts of the exact traveling wave solution of $\theta_{2,2}$ (b) the contour plot of the modulus (left), real (middle) and imaginary (right) parts of the exact traveling wave solution of $\theta_{2,2}(\mathbf{c})$ and 2D-polar plot of the modulus (left), real (middle) and imaginary (right) parts of the travelling wave solution of $\theta_{2,2}$ at $\mathrm{t}=0, \mathrm{t}=0.2, \mathrm{t}=0.4, \mathrm{t}=0.6, \mathrm{t}=0.8, \mathrm{t}=1$, respectively, when $p=1.5, q=1.5, a=1$, $\omega=2.5, b_{1}=-1, b_{2}=-0.5, \alpha=1.5, \beta=1, \varphi=1, v=1.5$ and $y=1$. 

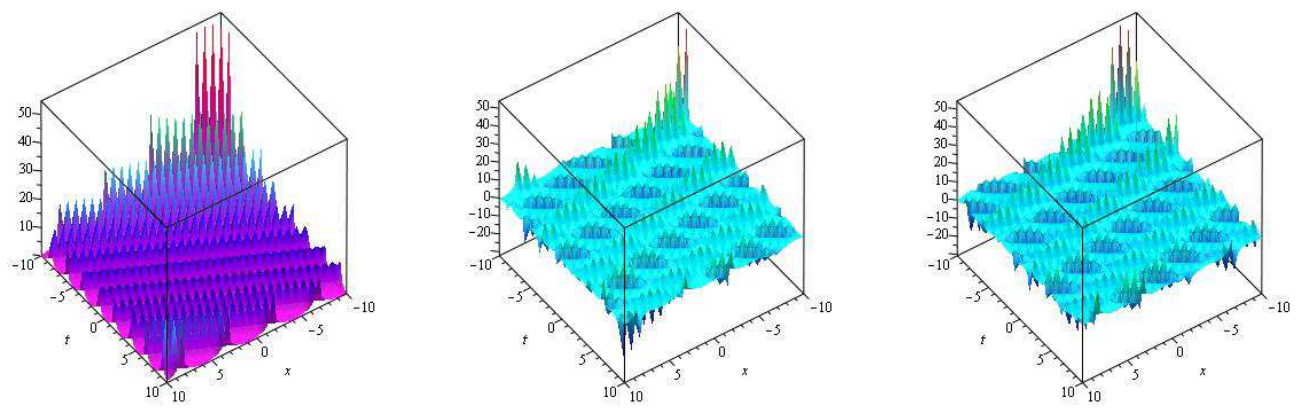

(a)

$\left|\theta_{3,1}\right|$

$\operatorname{Re}\left(\theta_{3,1}\right)$

$\operatorname{Im}\left(\theta_{3,1}\right)$
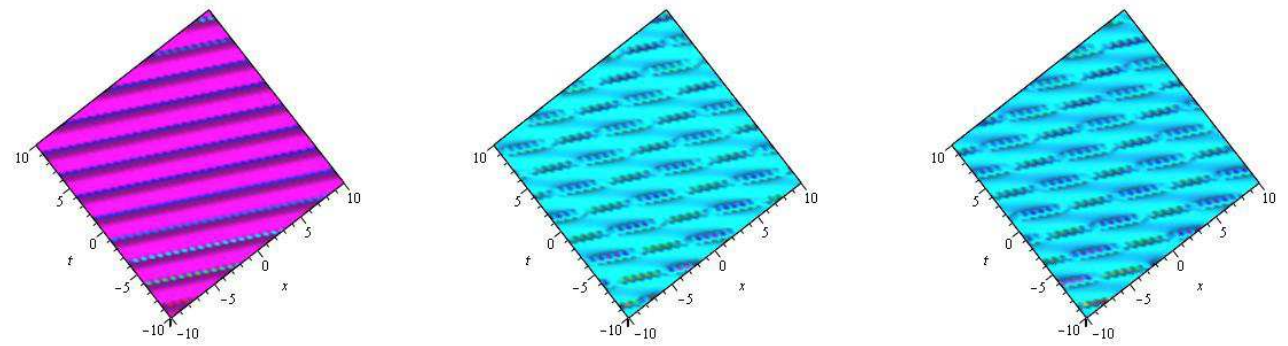

(b)

$\left|\theta_{3,1}\right|$

$\operatorname{Re}\left(\theta_{3,1}\right)$

$\operatorname{Im}\left(\theta_{3,1}\right)$
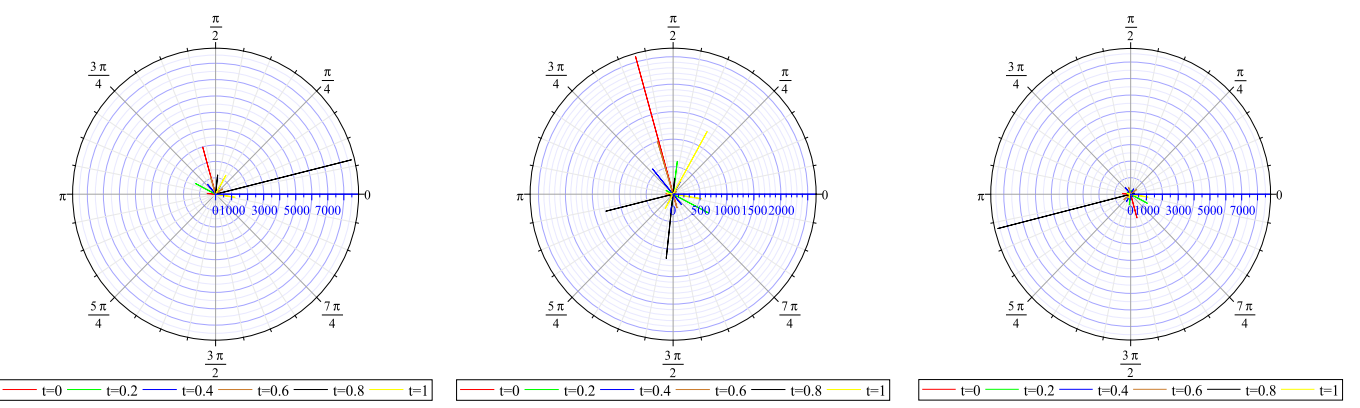

(c)

$$
\left|\theta_{3,1}\right|
$$

$$
\operatorname{Re}\left(\theta_{3,1}\right)
$$

$\operatorname{Im}\left(\theta_{3,1}\right)$

Figure 2. (a) 3D-plot of the modulus (left), real (middle) and imaginary (right) parts of the exact traveling wave solution of $\theta_{3,1}(\mathbf{b})$ the contour plot of the modulus (left), real (middle) and imaginary (right) parts of the exact traveling wave solution of $\theta_{3,1}$ (c) 2D-polar plot of the modulus (left), real (middle) and imaginary (right) parts of the travelling wave solution of $\theta_{3,1}$ at $\mathrm{t}=0, \mathrm{t}=0.2, \mathrm{t}=0.4, \mathrm{t}=0.6, \mathrm{t}=0.8, \mathrm{t}=1$, respectively, when $p=1.5, q=1, a=2.5$, $\omega=1.5, b_{1}=-3, b_{2}=-1, \alpha=0.5, \beta=1, \varphi=2, v=1$ and $y=1$. 

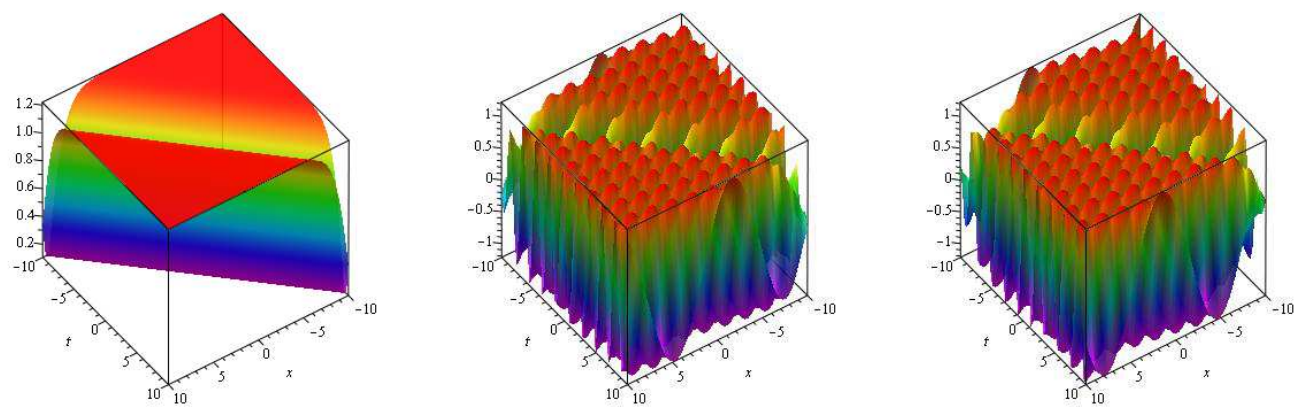

(a)

$$
\left|\theta_{5,1}\right|
$$

$\operatorname{Re}\left(\theta_{5,1}\right)$

$\operatorname{Im}\left(\theta_{5,1}\right)$
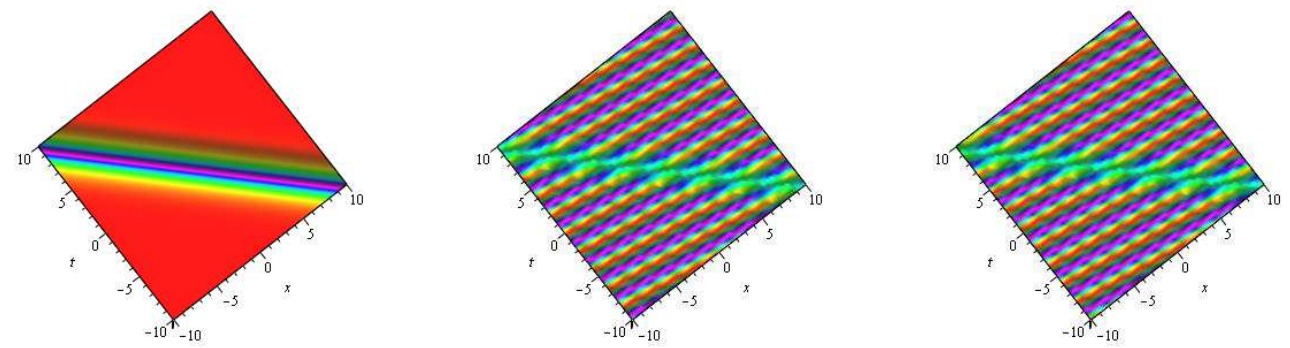

(b)

$\left|\theta_{5,1}\right|$

$\operatorname{Re}\left(\theta_{5,1}\right)$

$\operatorname{Im}\left(\theta_{5,1}\right)$
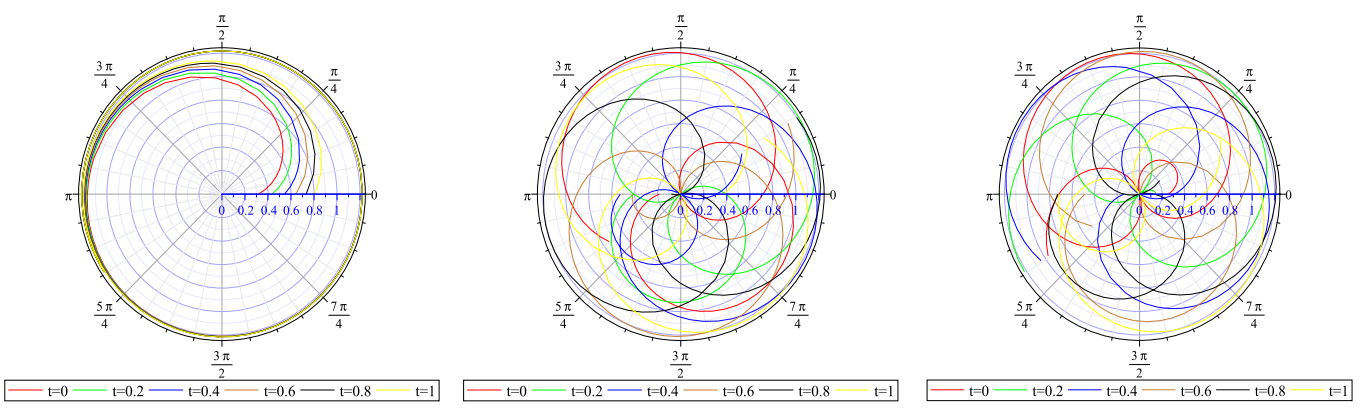

(c)

$$
\left|\theta_{5,1}\right|
$$

$\operatorname{Re}\left(\theta_{5,1}\right)$

$\operatorname{Im}\left(\theta_{5,1}\right)$

FiguRE 3. (a) 3D-plot of the modulus (left), real (middle) and imaginary (right) parts of the exact traveling wave solution of $\theta_{5,1}(\mathbf{b})$ the contour plot of the modulus (left), real (middle) and imaginary (right) parts of the travelling wave solution of $\theta_{5,1}(\mathbf{c}) 2 \mathrm{D}$-polar plot of the modulus (left), real (middle) and imaginary (right) parts of the exact traveling wave solution of $\theta_{5,1}$ at $\mathrm{t}=0, \mathrm{t}=0.2, \mathrm{t}=0.4, \mathrm{t}=0.6, \mathrm{t}=0.8, \mathrm{t}=1$, respectively, when $p=0.5, q=0.2, a=3$, $\omega=3, b_{1}=2, b_{2}=1.5, \alpha=2, \beta=1, \varphi=2, v=2$ and $y=1$. 

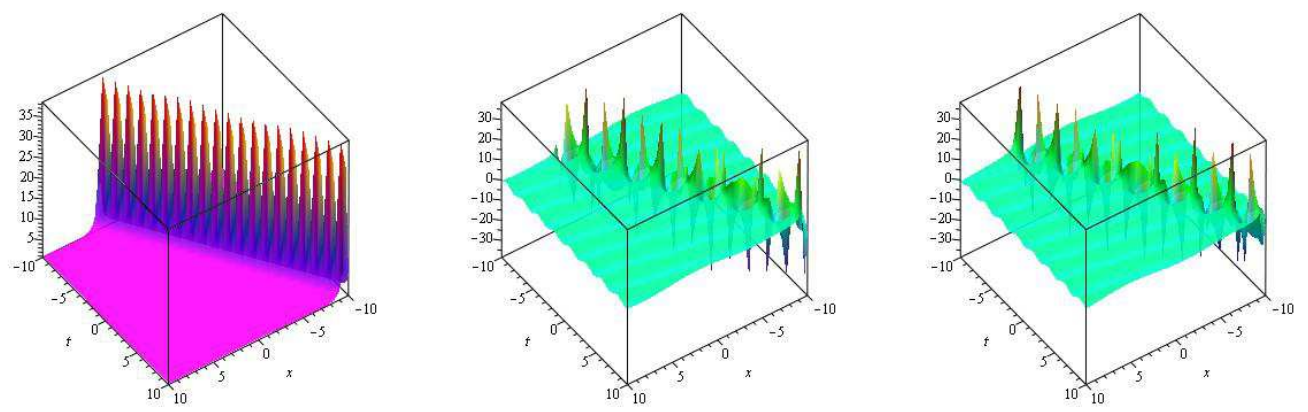

(a)

$$
\left|\theta_{8,3}\right|
$$

$\operatorname{Re}\left(\theta_{8,3}\right)$

$\operatorname{Im}\left(\theta_{8,3}\right)$
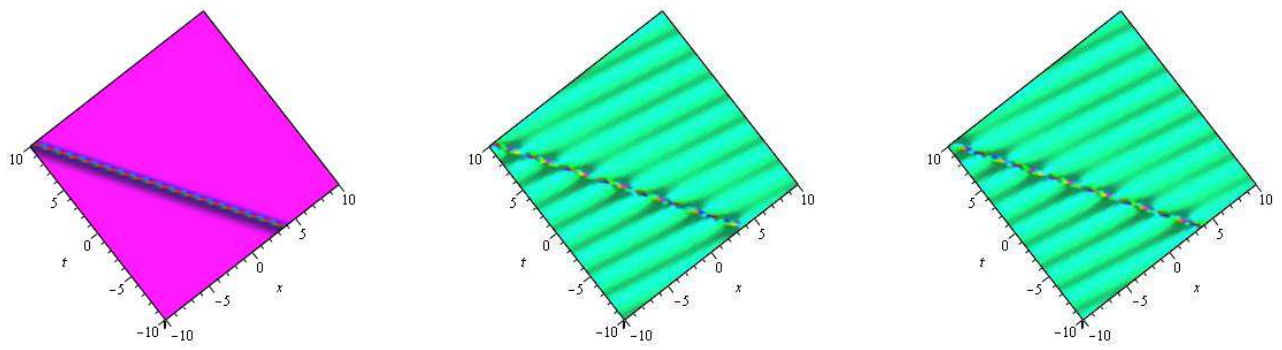

(b)

$\left|\theta_{8,3}\right|$

$\operatorname{Re}\left(\theta_{8,3}\right)$

$\operatorname{Im}\left(\theta_{8,3}\right)$
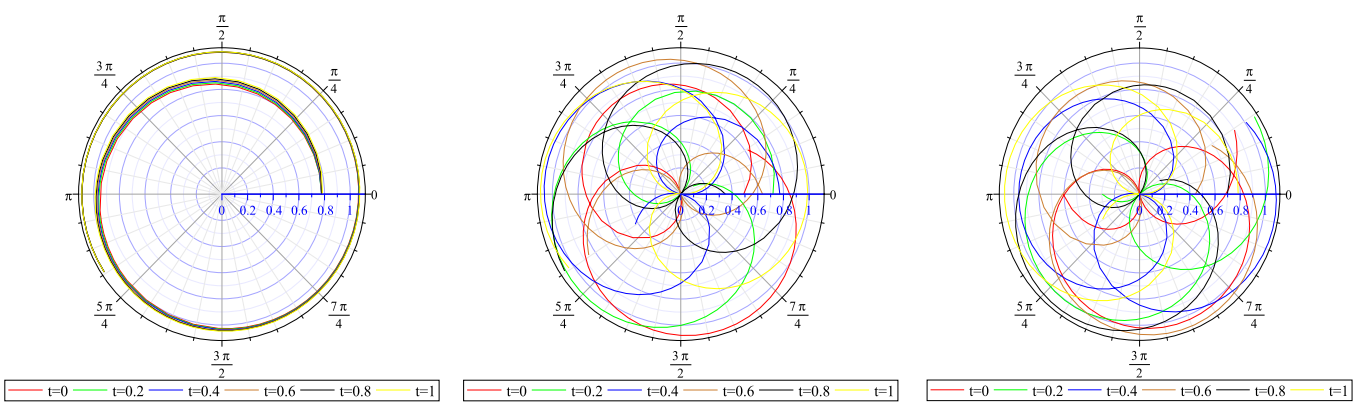

(c)

$\left|\theta_{8,3}\right|$

$\operatorname{Re}\left(\theta_{8,3}\right)$

$\operatorname{Im}\left(\theta_{8,3}\right)$

Figure 4. (a) 3D-plot of the modulus (left), real (middle) and imaginary (right) parts of the exact traveling wave solution of $\theta_{8,3}(\mathbf{b})$ the contour plot of the modulus (left), real (middle) and imaginary (right) parts of exact traveling wave solution of $\theta_{8,3}(\mathbf{c}) 2 \mathrm{D}$-polar plot of the modulus (left), real (middle) and imaginary (right) parts of the travelling wave solution of $\theta_{8,3}$ at $\mathrm{t}=0, \mathrm{t}=0.2, \mathrm{t}=0.4, \mathrm{t}=0.6, \mathrm{t}=0.8, \mathrm{t}=1$, respectively, when $p=0.5, q=0.75, a=1$, $\omega=2.5, b_{1}=2, b_{2}=0.5, \alpha=1.5, \beta=2, \varphi=1.5, v=1$ and $y=1$. 


$$
\Theta_{8,4}(x, y, t)=-\sqrt{\frac{a\left(p^{2}+q^{2}\right)+\omega}{2\left(p b_{1}+q b_{2}\right)}} \frac{\cosh \left[\sqrt{\frac{-2\left(a p^{2}+a q^{2}+\omega\right)}{a\left(\alpha^{2}+\beta^{2}\right)}}(\alpha x+\beta y-v t)\right]}{1-\sinh \left[\sqrt{\frac{-2\left(a p^{2}+a q^{2}+\omega\right)}{a\left(\alpha^{2}+\beta^{2}\right)}}(\alpha x+\beta y-v t)\right]} e^{i(p x+q y+\omega t+\varphi)} .
$$

\section{Graphichl Representations}

In this section, some of the graphical representations of the acquired exact traveling wave solutions to the CNLSE obtained by the extended rational sine-cosine/sinh-cosh techniques. The 3D plots for the modulus, real and imaginary parts, of the exact traveling wave solutions $\theta_{2,2}(x, t), \theta_{3,1}(x, t), \theta_{5,1}(x, t)$, and $\theta_{8,3}(x, t)$ are displayed in Figures 1(a), 2(a), 3(a) and 4(a), respectively. Figures 1(b), 2(b), 3(b) and 4(b), demonstrate the shape of contour plot of, indicated by the for the modulus, real and imaginary parts, of the exact traveling wave solutions $\theta_{2,2}(x, t), \theta_{3,1}(x, t), \theta_{5,1}(x, t)$ and $\theta_{8,3}(x, t)$. The 2D line plot of the modulus, real and imaginary parts, of the exact traveling wave solutions $\theta_{2,2}(x, t), \theta_{3,1}(x, t), \theta_{5,1}(x, t)$ and $\theta_{8,3}(x, t)$. are presented in Figures $1(\mathrm{c})$, $2(\mathrm{c}), 3(\mathrm{c})$ and $4(\mathrm{c})$ with $t=0, t=0.2, t=0.4, t=0.6, t=0.8, t=1$.

\section{Conclusion}

In this work, we have investigated diverse exact traveling wave solutions like hyperbolic and trigonometric solutions to (2+1)-dimensional CNLSE with extended rational sine-cosine and rational extended sinh-cosh schemes. These various kinds of solutions are favorable for explaining diverse nonlinear physical phenomena. Our results exhibited that the proposed methods are powerful and can be used to extract exact solutions for other NLPDEs that appear in many fields like engineering, mathematical biology, physics, chemistry, and vice versa. Furthermore, for the physical illustration of the acquired results, we plot 3D, 2D-polar and contour profiles by setting suitable values of involved parameters.

Acknowledgements. This research work has been supported by a research grant from the Amol University of Special Modern Technologies, Amol, Iran.

\section{REFERENCES}

[1] K.M. Abdul Al Woadud, D. Kumar, M.J. Islam, M. ImrulKayes and A.K. Joardar, Analytic solutions of the chiral nonlinear schrödinger equations investigated by an efficient approach. Int. J. Phys. Res. 7 (2019) 94-99.

[2] K.K. Ali, H. Rezazadeh, R.A. Talarposhti and A. Bekir, New soliton solutions for resonant nonlinear Schrödinger's equation having full nonlinearity. Int. J. Mod. Phys. B. 34 (2020) 2050032.

[3] S. Ali and M. Younis, Rogue wave solutions and modulation instability with variable coefficient and Harmonic potential. Front. Phys. 7 (2020) 255.

[4] A. Biswas, Perturbation of chiral solitons. Nucl. Phys. 806 (2009) 457-461.

[5] A. Biswas and D. Milovic, Chiral solitons with Bohm potential by He's variational principle. Phys. Atomic Nuclei. 74 (2011) $781-783$.

[6] A. Biswas, M.O. Al-Amr, H. Rezazadeh, Mirzazadeh, M. Eslami, Q. Zhou and M. Belic, Resonant optical solitons with dual-power law nonlinearity and fractional temporal evolution. Optik 165 (2018) 233-239.

[7] H. Bulut, T.A. Sulaiman and B. Demirdag, Dynamics of soliton solutions in the chiral nonlinear Schrödinger equations. Nonlinear Dyn. 91 (2017) 1985-1991.

[8] N. Cheema and M. Younis, New and more exact traveling wave solutions to integrable (2+1)-dimensional Maccari system. Nonlinear Dyn. 83 (2016) 1395-1401.

[9] M.T. Darvishi, M. Najafi and A. M. Wazwaz, New extended rational trigonometric methods and applications., Waves Random Comp. 30 (2018) 1-22.

[10] G. Ebadi, A. Yildirim and A. Biswas, Chiral solitons with bohm potential using $G^{\prime} / G$ method and exp function method. Rom. Rep. Phys. 64 (2012) 357-366.

[11] M.M.A. El-Sheikh, A.R. Seadawy, H.M. Ahmed, A.H. Arnous and W.B. Rabie, Dispersive and propagation of shallow water waves as a higher order nonlinear Boussinesq-like dynamical wave equations. Physica A. 537 (2020) 122662.

[12] M. Eslami, Trial solution technique to chiral nonlinear Schrödinger's equation in (1+2)-dimensions. Nonlinear Dyn. 85 (2016) $813-816$. 
[13] M. Eslami, M. Mirzazadeh and A. Biswas, Soliton solutions of the resonant nonlinear Schrödinger's equation in optical fibers with time dependent coefficients by simplest equation approach. J. Mod. Opt. 60 (2013) 1627-1636.

[14] F. Ferdousa, M.G. Hafeza, A. Biswasb, M. Ekicid, Q. Zhoue, M. Alfirasf, S.P. Moshokoac and M. Belic, Oblique resonant optical solitons with Kerr and parabolic law nonlinearities and fractional temporal evolution by generalized $\exp (-\phi(\xi))$-expansion. Optik 178 (2019) 439-448.

[15] W. Gao, M. Senel, G. Yel, H.M. Baskonus and B. Senel, New complex wave patterns to the electrical transmission line model arising in network system. Aims. Math. 5 (2020) 1881-1892.

[16] W. Gao, H. Rezazadeh, Z. Pinar, H.M. Baskonus, S. Sarwar and G. Yel, Novel explicit solutions for the nonlinear Zoomeron equation by using newly extended direct algebraic technique. Opt. Quant. Elect. 52 (2020) 1-13.

[17] D. Gianzo, J.O. Madsen, and J.S. Guilln, Integrable chiral theories in (2+1) dimensions, Nucl. Phys. B. 537 (1999) 586-598

[18] M. Iqbal, A.R. Seadawy, O.H. Khalil and D. Lu, Propagation of long internal waves in density stratified ocean for the (2+ 1)-dimensional nonlinear Nizhnik-Novikov-Vesselov dynamical equation. Res. Phys. 16 (2020) 102838.

[19] A. Javid and N. Raza, Chiral solitons of the $(1+2)$-dimensional nonlinear Schrodinger's equation. Mod. Phy. Lett. B. 33 (2019) 1950401.

[20] A.G. Johnpillai, A. Yildirim and A. Biswas, Chiral solitons with Bohm potential by lie group analysis and traveling wave hypothesis. Rom. J. Phys. 57 (2012) 545-554.

[21] A. Korkmaz, O.E. Hepson, K. Hosseini, H. Rezazadeh and M. Eslami, Sine-Gordon expansion method for exact solutions to conformable time fractional equations in RLW-class. J. King Saud Univ. Sci. 32 (2018) 567-574.

[22] D. Lu, A.R. Seadawy and M. Arshad, Applications of extended simple equation method on unstable nonlinear Shrodinger's equations. Optik 140 (2017) 136-144.

[23] N. Raza and A. Javid, Optical dark and dark-singular soliton solutions of (1+2)-dimensional chiral nonlinear Schrödinger's equation. Waves Random Complex. 29 (2018) 1-13.

[24] N. Raza, U. Afzal, A.R. Butt and H. Rezazadeh, Optical solitons in nematic liquid crystals with Kerr and parabolic law nonlinearities. Opt. Quant. Elect. 51 (2019) 107.

[25] N. Raza, M. Abdullah and A.R. Butt, Analytical soliton solutions of Biswas-Milovic equation in Kerr and non-Kerr law media. Optik 157 (2018) 993-1002.

[26] N. Raza, M. Abdullah, A.R. Butt, I.G. Murtaza and S. Sial, New exact periodic elliptic wave solutions for extended quantum Zakharov-Kuznetsov equation. Opt. Quant. Elect. 50 (2018) 177.

[27] N. Raza, M.R. Aslam and H. Rezazadeh, Analytical study of resonant optical solitons with variable coefficients in Kerr and non-Kerr law media. Opt Quant Elect. 51 (2019) 59.

[28] N. Raza and S. Arshed, Chiral bright and dark soliton solutions of Schrödinger's equation in (1+2)-dimensions. Ain. Shams. Eng. J. 11 (2020) 1237-1241.

[29] H.U. Rehman, M. Younis, S. Jafar, M. Tahir and M.S. Saleem, Optical Solitons of Biswas-Arshed Model in Birefrigent Fiber Without Four Wave Mixing. Optik 213 (2020) 164669.

[30] H. Rezazadeh, A. Korkmaz, M. Eslami and S.M. Mirhosseini-Alizamini, A large family of optical solutions to Kundu Eckhaus model by a new auxiliary equation method. Opt. Quant. Elect. 51 (2019) 84.

[31] K.U. Tariq, A.R. Seadawy, M. Younis and S.T.R. Rizv, Dispersive traveling wave solutions to the space-time fractional equal-width dynamical equation and its applications. Opt. Quant. Elect. 50 (2018) 147.

[32] H. Triki, R.T. Alqahtani, Q. Zhou and A. Biswas, New envelope solitons for Gerdjikov-Ivanov model in nonlinear optics. Superlattices Microstruct. 111 (2017) 326-334.

[33] B. Younas and M. Younis, Chirped solitons in optical monomode fibres modelled with Chen-Lee-Liu equation. Pramana - $J$ Phys. 94 (2020) 3.

[34] M. Younis, T.A. Sulaiman, M. Bilal, S.U. Rehman and U. Younas, Modulation instability analysis, optical and other solutions to the modified nonlinear Schrödinger equation. Commun. Theor. Phys. 72 (2020) 065001.

[35] M. Younis, M. Bilal, S.U. Rehman, U. Younas and S.T.R. Rizvi, Investigation of optical solitons in birefringent polarization preserving fibers with four-wave mixing effect. Int. J. Mod. Phys. B. 34 (2020) 2050113.

[36] M. Younis, U. Younas, S.U. Rehman, M. Bilal and A. Waheed, Optical bright-dark and Gaussian soliton with third order dispersion. Optik 134 (2017) 233--238.

[37] M. Younis, N. Cheema, S.A. Mahmood and S.T.R. Rizvi, On optical solitons: the chiral nonlinear Schrödinger equation with perturbation and Bohm potential. Opt. Quant. Elect. 48 (2016) 542. 